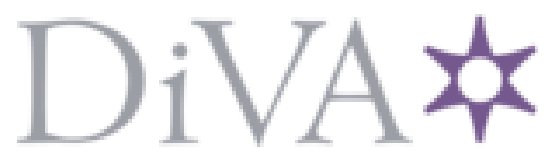

http://www.diva-portal.org

This is the published version of a paper presented at 2013 IEEE International Conference on Mechatronics and Automation (ICMA), 4-7 aug. 2013, Takamatsu, Japan.

Citation for the original published paper:

Albitar, H., Ananiev, A., Kalaykov, I. (2013)

New concept of in-water surface cleaning robot.

In: Mechatronics and Automation (ICMA), 2013 IEEE International Conference onDate 4-7 Aug.

2013 (pp. 1582-1587). IEEE conference proceedings

http://dx.doi.org/10.1109/ICMA.2013.6618150

N.B. When citing this work, cite the original published paper.

Permanent link to this version:

http://urn.kb.se/resolve?urn=urn:nbn:se:oru:diva-30340 


\title{
New concept of in-water surface cleaning robot
}

\author{
H. Albitar, A. Ananiev, I. Kalaykov \\ School of Science and Technology \\ Örebro University \\ 70182 Örebro, Sweden \\ \{houssam.albitar,anani.ananiev,ivan.kalaykov\}@oru.se
}

\begin{abstract}
This paper introduces a new concept of flexible crawling mechanism to design an industrial underwater cleaning robot, which is evaluated from the viewpoint of the capability to work underwater, scanning the desired surface, and bearing the reactions. This can be used as a robotic application in underwater surface cleaning and maintenance. We designed a robot that realizes the motion by contraction and extraction using DC-motors and vacuum technology. In this study we first focused on realizing the adhesion, bearing reactions, and achieving a stable locomotion on the surface.
\end{abstract}

\section{INTRODUCTION}

Bio-fouling buildup material on the in-water surfaces of structures, such as petroleum and gas storage tanks, electric power plants, ships, bridges, oil rigs, etc., creates a number of problems:

- worse roughness and less hydrodynamic surface caused by hard and soft bio-fouling formations;

- increased energy and fuel consumption by vessels with bio-fouled hulls (> 25 - 30\% [1]);

- increase in frictional drag or decrease of operational efficiency in cooling systems, e.g. in power plants or other installations [2];

- intensive corrosion of in-water structures fouled with seaweed and barnacles [3], [4];

- restricted water flow and deteriorated heat-transfer in the heat exchangers (clogging) in cooling systems using sea water (nuclear power plant cooling tunnels, oil rigs water lines) [5].

Underwater operations related to effective management of bio-fouling and other contaminants, including effective capture of biological material, present unique challenges for robotic applications. Humans are limited in in-water missions in duration and depth [6], therefore using robotic systems in in-water environment is very effortful due to limited visibility, hazardous surrounding and external forces applied to the robot.

Methods for in-water ship cleaning and assessing the risks associated with it are discussed in [1]. Among several options of surface cleaning tools, we selected water jets to be used, as they naturally fit the in-water environment. Advantage is their relative simplicity, reliability and easiness in setting various regimes according the type of surface paint and type of fouling (soft or hard). They can be simply attached to the cleaning robot at a non-disturbing the locomotion place on the robot construction, explained in the next section.The main drawback of water jets is the created considerable reaction forces that has to be balanced by the adhesion subsystem or by additional reaction forces neutralizing subsystem if adhesion is not enough. In this paper we don't discuss the cleaning tools in details.

In this paper, a new concept of crawling in-water surface cleaning robot is presented in Section II. Main focus is at the locomotion presented in Section III. Section IV presents how we keep the robot on the surface to be cleaned and neutralize the reactions of the cleaning forces. Conclusions and future work are outlined in Section V.

\section{IN-WATER CLEANING ROBOT CONCEPT}

Usually surface cleaning takes place in smooth flat 2Dspace, therefore the cleaning robot needs minimum two active (controllable) degrees of freedom (2DOF). There exist huge amount of mobile robots providing 2D movements. Not many of them are able to operate in in-water environment for obvious reasons, the major of which is the necessity to work on vertical or negatively inclined surfaces. Therefore dedicated means to compensate the gravity and to adapt the robot to the surface profile are needed, for example additional passive DOFs to accommodate the robot's structure to the concavity/convexity of the surface (Fig. 1).
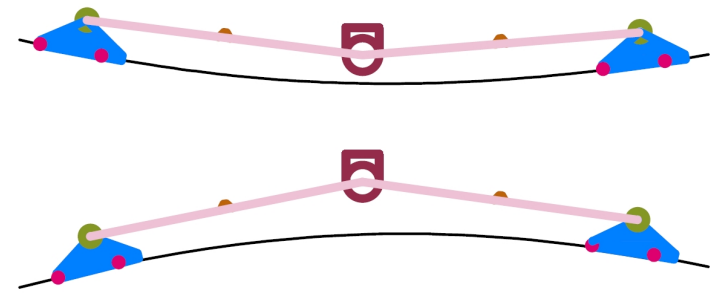

Fig. 1: Robot adapting to surface profile

In our concept, we propose an in-water crawling robot (Fig. 2) having four main subsystems, namely:

- locomotion,

- cleaning,

- adhesion, and

- neutralization of reaction forces.

The locomotion subsystem is the basic component providing all movements of the robot. Its kinematics is described in details in this section.

We selected the crawling mechanism because it: 
- it adapts to the surface (almost plane without sharp edges, Fig. 1);

- it can be used for different types of surface material;

- it is a simple mechanism;

- it gives stable low center of gravity and large base support in cleaning cycle.

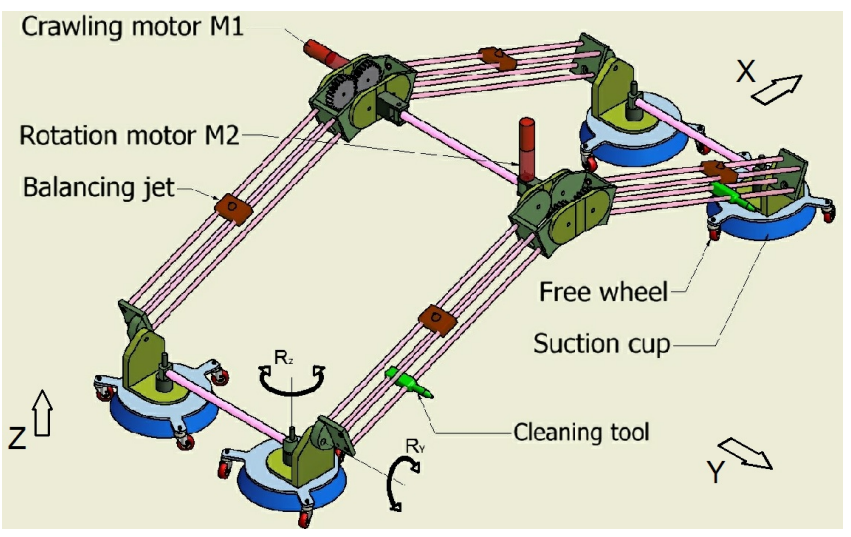

Fig. 2: The cleaning robot configuration

This robot consists of seven links connected through six joints marked by a, b, c, d, e, f on Fig. 3. Links (a-e), (e-d), (b-f) and (f-c) are constructed by four parallel lightweight bars for achieving stiffness. Each joint has two rotational degrees of freedom $\mathcal{R}_{Y}$ and $\mathcal{R}_{Z}$ shown on one joint on Fig.2. Joint $\mathbf{f}$ is actuated by motor $\mathbf{M 1}$ for $\mathcal{R}_{Y}$ rotation around $\mathbf{Y}$ axis, and joint $\mathbf{e}$ is actuated by motor $\mathbf{M 2}$ for $\mathcal{R}_{Z}$ rotation around $\mathbf{Z}$ axis. The rest degrees of freedom $\mathbf{a}, \mathbf{b}, \mathbf{c}, \mathbf{d}$ are passive in both $\mathcal{R}_{Y}$ and $\mathcal{R}_{Z}$ DOFs.

Four suction cups marked by $\mathbf{A}, \mathbf{B}, \mathbf{C}, \mathbf{D}$ are attached correspondingly at the four corner joints $\mathbf{a}, \mathbf{b}, \mathbf{c}, \mathbf{d}$. Each cup is surrounded by three small free wheels to minimize the friction of movement when any of these cups is not enabled (OFF-state).

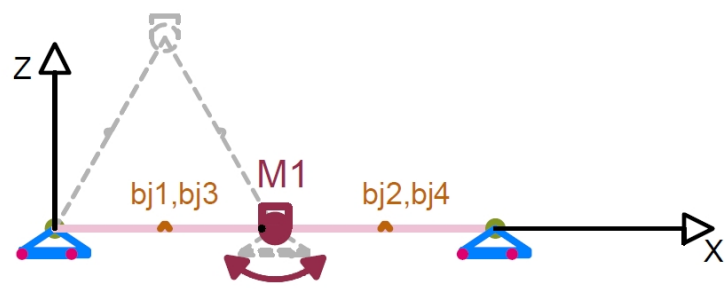

I.Side view

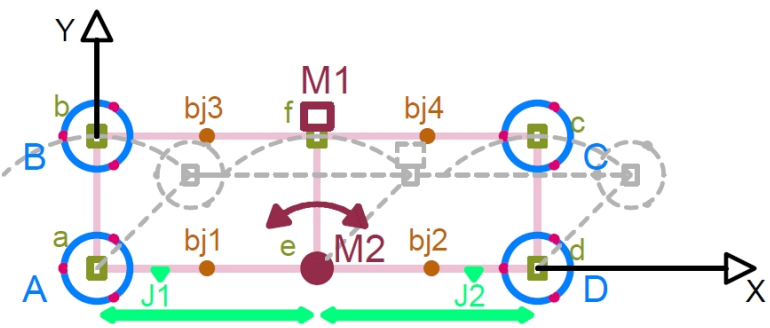

\section{Top view}

Fig. 3: Simplified presentation of the robot
Links (a-e) and (e-d) plus cups $\mathbf{A}$ and $\mathbf{D}$ form a biped arrangement (a-e-d), while links (b-f) and (f-c) plus cups B and $\mathbf{C}$ form another parallel biped arrangement (b-f-c). These two bipeds connected with three parallel links (a-b), (e-f), (c-d) materialize the crawling mechanism of the robot. The crawling is achieved by motor $\mathbf{M 1}$ around $\mathcal{R}_{Y}$. Figure 3, top, represents side view of max-span and intermediate (dashed) configurations. The suction cups adjust to the surface via the passive $\mathcal{R}_{Y}$ DOFs. In this example, the cups stay horizontal regardless the angle between the biped legs.

At the same time, both bipeds with the connecting links $(\mathbf{a}-\mathbf{b}),(\mathbf{e}-\mathbf{f}),(\mathbf{c}-\mathbf{d})$ form two parallelograms (a-b-f-e) and (ef-c-d) as shown on Fig. 3, top view, at max-span of the bipeds. The three links (a-b), (e-f), (c-d) are parallel in all possible configurations. When joint $\mathbf{e}$ is actuated by $\mathbf{M} 2$, the rest joints passively follow the rotation around $\mathcal{R}_{Z}$ as shown by dashed lines on Fig. 3 and further on Fig. 5 and Fig. 6.

With this arrangement the robot has two front sides and can move in both $X$ and $Y$ directions and to rotate around any cup. In all figures we assume (C-D) cups are at the $X$ axis front side and (A-D) cups are at the $Y$-axis front. The selection of front sides is arbitrary and obviously front and rear sides are interchangeable.

In fact, the basic movement is in $X$ direction, but combining it with rotation the robot can achieve the additional movement in $Y$ direction. Details about the locomotion are given in next Section III.

Briefly said, the cleaning subsystem contains two water jets directed on controllable varying angle to the surface. They are installed on two of the four bars shown on Fig.2 on the two links (a-e) and (e-d) on Fig. 3. The jets slide on these two bars and a separate control system regulates the linear speed and time of cleaning in accordance to the nature of bio-fouling. A vision camera provides feedback to the human operator for the cleaning quality.

For obvious reasons, the robot is tethered to external equipment providing human-machine interface to operator and power for motion and mainly for cleaning purposes.

The functional flexibility and the simplicity are the main features that characterize this concept in contrast to other similar solutions. The proposed robot:

- Has the minimum DOFs needed for moving onto a nonflat 2D space without obstacles, namely only two DOFs in comparison to four DOFs in RAMR I [7] and Alicia3 [8], five DOFs in MATS [9], six DOFs in ROMA II [7], consequently, the control system is simpler [10].

- Suits well for in-water environment in terms of the nature of the adhesion and the use of water for cleaning, contrarily to many out-of-water solutions like [11], [12].

- Can perform the cleaning on moving surface such as ship hull during its cruise in the ocean.

- Can neutralize the force reactions resulting from the cleaning process in contrast to the similar robots that are limited mainly for inspection (ROMA II [7]), welding [13] or for cleaning flat surfaces (Sky Cleaner 1,2,3 [14], NINJA [15]). 
- Has "pseudo-holonomic" property brought by the movement in $Y$ direction orthogonal to $X$ or movement to any other direction after rotation.

\section{ROBOT LOCOMOTION}

The two active degrees of freedom $\mathcal{R}_{Y}$ and $\mathcal{R}_{Z}$ actuated by motors $\mathbf{M 1}$ and $\mathbf{M} 2$ respectively have to be combined with ON/OFF activation of the suction cups in order robot to move. Enabled suction at one place and disabled suction at others gives the needed freedom of robot to move by activating M1 and M2 in coordinated way with suction cups' activation. By special control sequences the robot can perform three basic movements:

- crawling movement (on $X$-axis),

- rotation (around $Z$-axis),

- stepping forward (on $Y$-axis).

Combining these movements the robot can scan the entire 2D space to be cleaned. Next subsections describe in details how they are implemented.

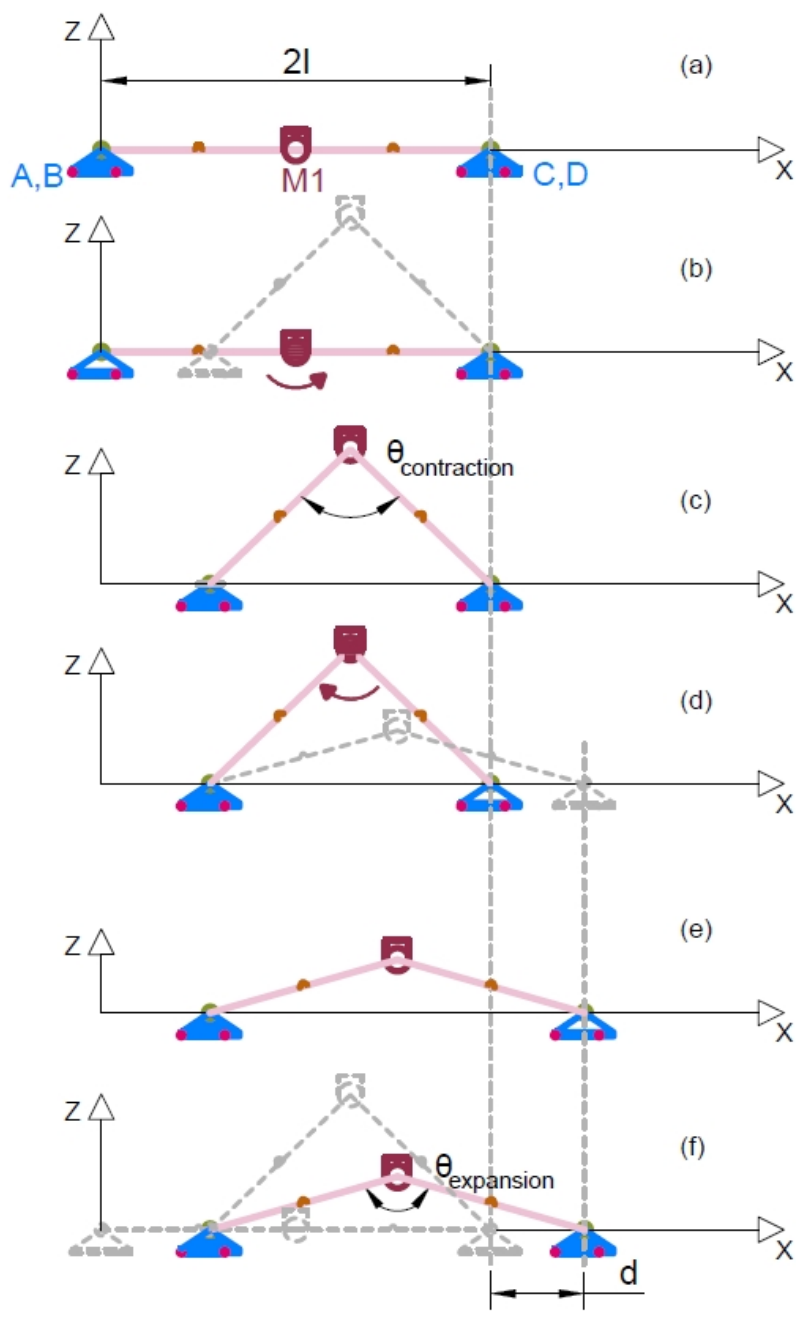

Fig. 4: Crawling movement sequence

\section{A. Crawling movement (on $X$-axis)}

The crawling movement is illustrated on Fig. 4, where initial position is at Fig.4(a) and final position is at Fig.4(f).
Without loss of generality, it is assumed the bipeds are at max-span pose (angle between the legs is $180^{\circ}$ ) at initial position. The process of moving straight forward starts with switching OFF the rear pair of suction cups (A-B), Fig.4(b), and starting the crawling motor M1 to initiate contracting motion of both bipeds, Fig.4(c). After desired angle $\theta_{\text {contraction }}$ is achieved, motor $\mathbf{M 1}$ is stopped and the (A-B) pair is switched ON again, Fig.4(c). The expanding motion is done by switching OFF the front cups (C-D), Fig.4(d), then motor M1 rotates in the opposite direction. After a desired angle $\theta_{\text {expansion }}$ is achieved, motor M1 is stopped, Fig.4(e), and the (C-D) pair is switched ON again, Fig.4(f) - the robot is fixed at the final position. The crawled distance $d$ is computed from:

$$
d=2 l\left[\sin \left(\theta_{\text {expansion }} / 2\right)-\sin \left(\theta_{\text {contraction }} / 2\right)\right],
$$

where $l$ is the robot links length. By using any values of $\theta_{\text {contraction }}, \theta_{\text {expansion }}$ limited between $30^{\circ}$ and $180^{\circ}$ due to construction limitations, any desired distance in the range $[0, l]$ can be implemented.

The control sequence is:

1) Switch OFF rear cups (A-B), hold front cups (C-D).

2) Activate $\mathbf{M 1}$ counterclockwise to angle $\theta_{\text {contraction }}$.

3) Switch ON rear cups (A-B).

4) Switch OFF front cups (C-D), hold rear cups (A-B).

5) Activate M1 clockwise on angle $\theta_{\text {expansion }}$.

For moving straight backward the same control sequence is used, in which the front and rear cups swap their places and the rotation of $\mathbf{M 1}$ is in the respective opposite direction.

During the described movement, when any of the suction cups is OFF, there is a need to keep the robot in contact with the surface because the suction cups have passive DOF around $\mathcal{R}_{Y}$. Therefore, in the course of motion neutralizing (balancing) water jets marked by (bj1, bj2, bj3 and bj4) on Fig. 3 are activated. Details about these jets are given in next section. The small free wheels around the suction cups minimize the friction to the surface at contraction and expansion phases.

For achieving maximum production rate, the cleaning operation is performed when the robot is immobilized into its max-span pose - all suction cups are ON and balancing jets are activated. This provides the center of robot's mass close to the surface, which means maximum stability.

Assuming $\omega_{M 1}$ is the angular speed of the bipeds shaft driven by $\mathbf{M} 1$ and $t_{s}$ is the suction cups switching time, the time of one contraction+expansion cycle is:

$$
T_{c r}=\left(\theta_{\text {contraction }}+\theta_{\text {expansion }}\right) / \omega_{M 1}+4 t_{s} .
$$

Thene the linear speed of crawling is:

$$
V_{c r}=\frac{d}{T_{c r}}=\frac{2 l\left[\sin \left(\theta_{\text {expansion }} / 2\right)-\sin \left(\theta_{\text {contraction }} / 2\right)\right]}{\left(\theta_{\text {contraction }}+\theta_{\text {expansion }}\right) / \omega_{M 1}+4 t_{s}} .
$$

\section{B. Changing direction (rotation around $Z$-axis)}

While crawling is implemented by rotation around $Y$-axis, the changing of direction is done by rotation around $Z$-axis. It can be realized around the center of any suction cup, i.e. 
there are four possible centers of rotation. Similar approach of applying a control sequence of activating/deactivating motor $\mathbf{M} 2$ and the suction cups.

Let's consider that robot is to rotate around suction cup A clockwise on angle $\alpha,|\alpha| \leq 60^{\circ}$, top view of the robot shown on Fig. 5. At the initial pose all suction cups are ON, the bipeds are a max-span for better understanding. In fact, the bipeds could have any vertical fixed by motor M1. The rotation is initiated by switching OFF suction cups (B-C) and starting motor M2, Fig. 5(b). After the desired angle $\alpha$ is achieved, motor M2 is stopped and cups (B-C) are switched ON again. The robot has an intermediate pose given on Fig. 5(c). After that cups (C-D) are switched OFF and motor M2 rotates in the opposite direction, Fig.5(d). After the desired angle $\alpha$ is reached, $\mathbf{M 2}$ is stopped and the pair (C-D) is switched ON again - the robot is at the final position, Fig.5(e).

The control sequence (for clockwise rotation around suction cup A) is:

1) Switch OFF cups (B-C), hold front cups (A-D).

2) Activate $\mathbf{M} 2$ clockwise to angle $\alpha$.

3) Switch ON cups (B-C).

4) Switch OFF cups (C-D), hold cups (A-B).

5) Activate $\mathbf{M} 2$ counterclockwise on angle $\alpha$.

Similar sequence can be easily derived for any cup.

As a rule for this construction: rotation around any cup requires it to stay always ON during the movement. Similarly to crawling, during change of direction the balancing jets are activated when any suction cup is OFF, as they all have passive DOFs around $\mathcal{R}_{Z}$.

The coordinates of the robot's center from initial point G0 first moves to point $\mathbf{G 1}$ computed by:

$$
\left[\begin{array}{l}
x_{G 1} \\
y_{G 1}
\end{array}\right]=\left[\begin{array}{ll}
1 & \sin \alpha \\
0 & \cos \alpha
\end{array}\right] \cdot\left[\begin{array}{l}
x_{G 0} \\
y_{G 0}
\end{array}\right],
$$

then to $\mathbf{G 2}$ computed by:

$$
\left[\begin{array}{l}
x_{G 2} \\
y_{G 2}
\end{array}\right]=\left[\begin{array}{cc}
\cos \alpha & \sin \alpha \\
-\sin \alpha & \cos \alpha
\end{array}\right] \cdot\left[\begin{array}{l}
x_{G 0} \\
y_{G 0}
\end{array}\right],
$$

obtained by standard coordinate transformations. These equations are to be used in the robot path planning.

Assuming $\omega_{M 2}$ is the angular speed of shaft driven by M2, the time of changing direction (rotation) on angle $\alpha$ is:

$$
T_{\text {rot }}=2 \alpha / \omega_{M 2}+4 t_{s} .
$$

Then the angular speed of changing direction (rotation) on angle $\alpha$ is:

$$
\Omega_{\text {rot }}=\frac{\alpha}{T_{\text {rot }}}=\frac{\alpha}{2 \alpha / \omega_{M 2}+4 t_{s}} .
$$

\section{Stepping forward (on $Y$-axis)}

This basic movement is implemented by applying the same strategy of changing direction. In fact, we can do it as a consecutive execution of four rotations around $Z$-axis - one around cup $\mathbf{A}$ and one around cup $\mathbf{C}$ as shown on Fig. 6. For the first rotation suction cups (B-C) are switched OFF and motor M2 is activated clockwise to accomplish angle $+\beta$,
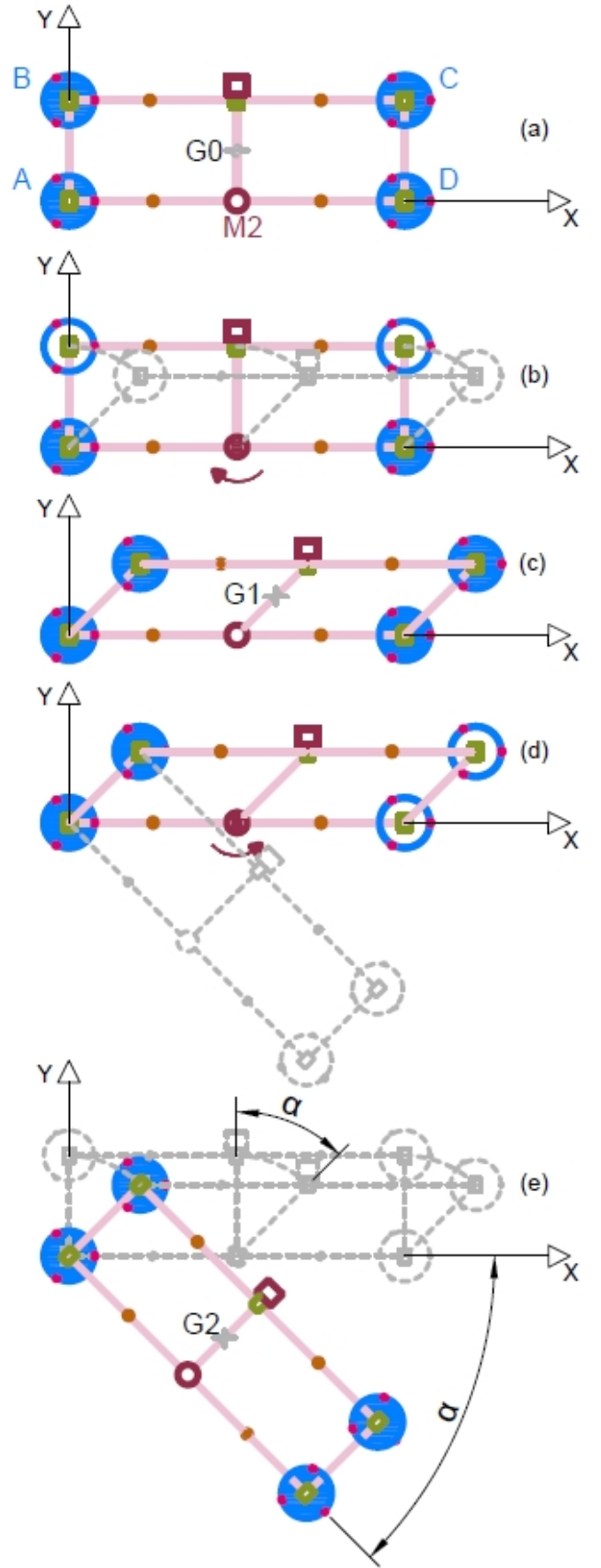

Fig. 5: Changing direction (rotation around cup A)

Fig. 6(b). Then cups (B-C) are switched ON to finalize the rotation around $\mathbf{A}$. The second rotation starts with putting cups (A-D) in OFF followed by activating M2 to rotate counter clockwise on angle $-\beta$, Fig. 6(c). At the end of this rotation cups (A-D) are switched ON. The third rotation starts with putting cups (B-C) in OFF and activating M2 counter clockwise on angle $-\beta$, Fig. $6(\mathrm{~d})$. Then (B-C) are switched ON and (A-D) are OFF and M2 is activated to rotate clockwise on angle $\beta$, Fig. 6(e). At the end all cups are switched ON - the robot reached final position, Fig.6(f).

One step forward $s$ on $Y$-axis depends on the angle $\beta$ :

$$
s=2 h(1-\cos \beta),
$$



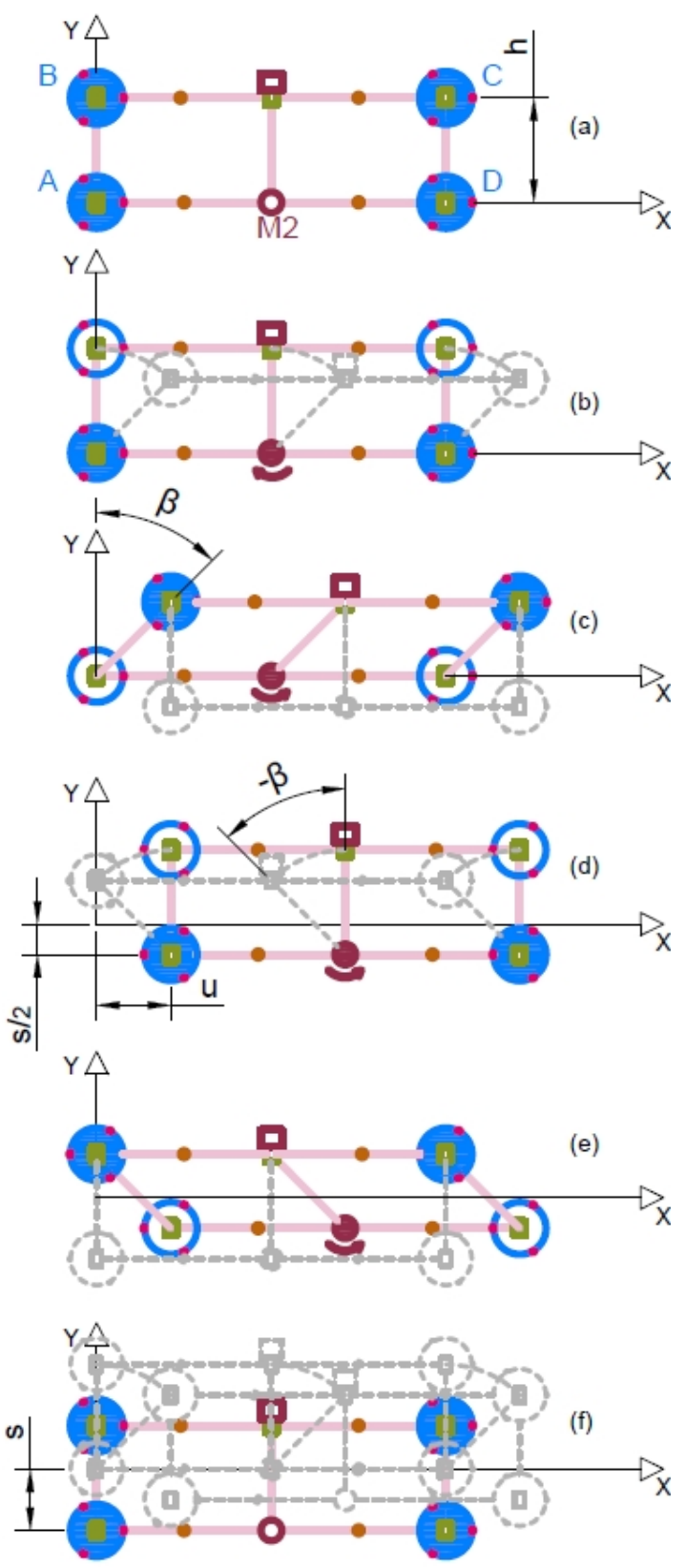

Fig. 6: stepping forwards

where $h$ is the length of the parallel links $(\mathbf{A}-\mathbf{B}),(\mathbf{E}-\mathbf{F})$ and (C-D). Assuming same meaning of $\omega_{M 2}$ and $t_{s}$ as above, the time of one step forward is:

$$
T_{\text {step }}=4\left(\beta / \omega+2 t_{s}\right)
$$

Then the linear speed of stepping forward on $Y$-axis is:

$$
V_{\text {step }}=\frac{s}{T_{\text {step }}}=\frac{h(1-\cos \beta)}{2\left(\beta / \omega_{M 2}+2 t_{s}\right)} .
$$

\section{ADHESION AND NEUTRALIZING SUBSYSTEMS}

\section{A. Adhesion by suction cups}

An important part of the proposed in-water robot is the adhesion mechanism that preserves the physical contact of the cleaning platform to the surface without sacrificing mobility. In the project, the analysis was focused at various adhesion principles and techniques such as suction type (e.g. [7], [16]), grasping type (e.g. [17]), magnetic type and biomimetic type. Magnetic type adhesion (e.g. [18]) obviously can work only on ferromagnetic surfaces, hence it is not applicable for concrete, plastic, wooden, etc. in-water surfaces. Biomimetic type adhesion (e.g. based on elastomers [19], gecko inspired feet [20], directional adhesion [21] and others [10]) is still in experimental phase with proven functionality for small sizes and weights, yet not proven for in-water application.

Due to the limitations of most of them we selected adhesion by suction cups as they are light weight, have simple control and allow movement over arbitrarily surfaces of various materials This type of adhesion is well developed and commercial. Of course, an external hydraulic generator connected to the robot by a hose in the tether link produces the under-pressure.

\section{B. Neutralizing subsystem}

In the cleaning subsystem, the force of the water being discharged from the jet nozzle creates an equal and opposite reaction, which makes the nozzle recoil in the opposite direction of the water flow [22], [23], [24]. This effect becomes greater as water flow increases.

The cleaning water jets are directed at certain angle to the surface, therefore the generated reaction force drags the robot away from the surface as shown of Fig. 7. For compensating and neutralizing the reaction force four dedicated waters jets (bj1, bj2, bj3, bj4) are installed on the four links (A-E), (E-D), (B-F), (F-D) on Fig. 2 and Fig. 3. While robot is in contact to the surface, thrust forces generated by these jets reinforce the adhesion.

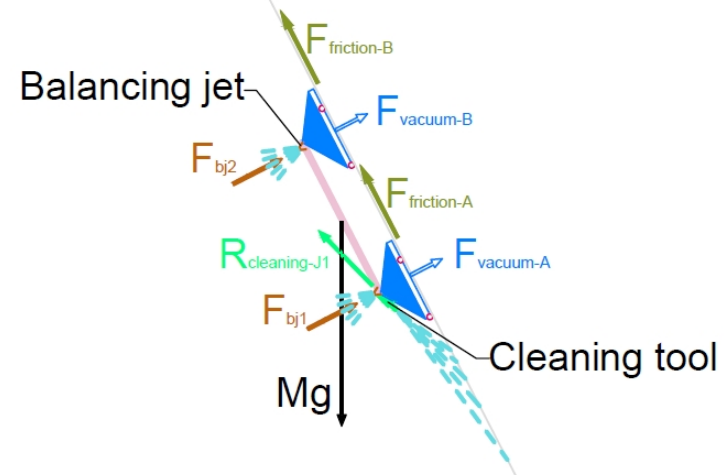

Fig. 7: Compensating the cleaning jets' reaction 
The water flow through the balancing jets is regulated such that the vector sum of all forces is nullified (Fig. 7) according

$$
\overrightarrow{M g}+\overrightarrow{F_{\text {vacuum }}}+\overrightarrow{F_{\text {friction }}}+\overrightarrow{F_{\text {bj }}}+\overrightarrow{R_{\text {cleaning }}}=0,
$$

where $\overrightarrow{M g}$ is the gravity force, $\overrightarrow{F_{\text {vacuum }}}$ is the force created by the suction, $\overrightarrow{F_{\text {friction }}}$ is the friction force at the contact, $\overrightarrow{F_{b j}}$ is the thrust force created by the balancing jets and $\overrightarrow{R_{\text {cleaning }}}$ is the thrust force created by the cleaning jet. For simplicity only two of the four balancing jets and only one cleaning jet are marked on Fig. 7. Same condition has to be fulfilled to achieve stable contact of the cleaning robot during cleaning phase.

\section{CONCLUSIONS AND FUTURE WORK}

A new concept of crawling robot for in-water cleaning of surfaces is presented in this paper. The design is based on minimalistic approach to achieve a compromise between maximum performance and reasonable complexity and cost of the system. The system description here is limited only to the kinematic structure and main functionalities of the robot, as it is still in a process of prototyping.

The solution is characterized by using minimum active DOFs (only two) for performing movements practically in all possible directions on a non-flat $2 \mathrm{D}$ surface. The available passive DOFs provide adaptability to local concavity/convexity of the surface to be cleaned.

The robot is aimed mainly to clean bio-fouled in-water surfaces, however it kinematics allows the execution of many other out-of-water tasks with respective change of the adhesion and neutralizing subsystems - from water-based to air-based.

\section{REFERENCES}

[1] J. Bohlander, "Review of options for in-water cleaning of ships," tech. rep., Ministry of Agriculture and Forestry, New Zealand, 2009.

[2] T. R. Bott, Fouling of heat exchangers, ch. 1.6, pp. 46-57. Elsevier Science, 1995.

[3] A. Iborra, J. Pastor, D. Alonso, B. Alvarez, F. Ortiz, P. Navarro, C. Fernández, and J. Suardiaz, "A cost-effective robotic solution for the cleaning of ships' hulls," Robotica, vol. 28, pp. 453-464, May 2010.

[4] A. H. Taylor and G. Rigby, "The identification and management of vessel biofouling areas as pathways for the introduction of unwanted aquatic organisms," tech. rep., Alan H. Taylor \& Asssociates, Australia, 2002.

[5] K. Satpathy, A. Mohanty, G. Sahu, S. Biswas, M. Prasad, and M. Slvanayagam, "Biofouling and its control in seawater cooled power plant cooling water system - a review," in Nuclear Power, pp. 191-242, InTech, 2010.

[6] T. Akinfijevs, A. Januševskis, and E. Lavendelis, "A Brief Survey of Ship Hull Cleaning Devices," Transport and Engineering, pp. 133146, 2007.

[7] C. Balaguer, A. Gimenez, and M.Abderrahim, "ROMA robots for inspection of steel based infrastructures," Industrial Robot: An International Journal, vol. 29, no. 3, pp. 246-251, 2002.

[8] D. Longo and G. Muscato, "The Alicia3 Climbing Robot," IEEE Robotics and Automation Magazine, no. 2, pp. 32-41, 2006.

[9] G. Bolmsjö, M. Olsson, and U. Lorentzon, "Development of a general purpose robot arm for use by disabled and elderly at home," 7-11 October 2002.

[10] B. Chu, K. Jung, C.-S. Han, and D. Hong, "A survey of climbing robots: Locomotion and adhesion," International Journal of Precision Engineering and Manufacturing, vol. 11, no. 4, pp. 633-647, 2010.
[11] C. Fernandez-Andres, A. Iborra, B. Alvarez, J. Pastor, P. Sanchez, J. Fernandez-Merono, and N. Ortega, "Ship shape in Europe: cooperative robots in the ship repair industry," Robotics Automation Magazine, IEEE, vol. 12, pp. 65-77, Sept. Sept.

[12] P. Navarro, J. Suardiaz, P. Alcover, R. Borraz, A. Mateo, and A. Iborra, "Teleoperated Visual Inspection System for Hull Spot-Blasting," in IEEE Industrial Electronics, IECON 2006 - 32nd Annual Conference on, pp. 3845-3850, Nov.

[13] M. Armada, M. Prieto, T. Akinfiev, R. Fernández, P. González, E. García, H. Montes, H. Montes, R. Ponticelli, J. Sarria, J. Estremera, S. Ros, J. Grieco, and G. Fernandez, "On the design and development of climbing and walking robots for the maritime industries," Journal of Maritime Research, vol. 2, no. 1, pp. 9-32, 2005.

[14] H. Zhang and J. Zhang, "A series of pneumatic glass-wall cleaning robots for high-rise buildings," Industrial Robot: An International Journal, vol. 32, no. 2, pp. 150-160, 2007.

[15] A. Nagakubo and S. Hirose, "Walking and running of the quadruped wall-climbing robot," in Robotics and Automation, 1994. Proceedings., 1994 IEEE International Conference on, pp. 1005-1012 vol.2, May.

[16] D. Longo and G. Muskato, "A Modular Approach for the Design of the Alicia3 Climbing Robot for Industrial Inspection," Industrial Robot: An International Robot, vol. 31, no. 2, pp. 148-158, 2004.

[17] H. Zhang, J. Zhang, R. Liu, and G. Zong, "Climbing technique of the cleaning robot for a spherical surface," in Mechatronics and Automation, 2005 IEEE International Conference, vol. 4, pp. 20612066 Vol. 4, July-1 Aug.

[18] F. Ortiz, J. Pastor, B. Alvarez, A. Iborra, N. Ortega, D. Rodriguez, and C. Concsa, "Robots for hull ship cleaning," in Industrial Electronics, 2007. ISIE 2007. IEEE International Symposium on, pp. 2077-2082, June 2007.

[19] O. Unver and M. Sitti, "A miniature ceiling walking robot with flat tacky elastomeric footpads," in Robotics and Automation, 2009. ICRA '09. IEEE International Conference on, pp. 2276-2281, May.

[20] C. Menon, M. Murphy, and M. Sitti, "Gecko Inspired Surface Climbing Robots," in Robotics and Biomimetics, 2004. ROBIO 2004. IEEE International Conference on, pp. 431-436, Aug.

[21] S. Kim, M. Spenko, S. Trujillo, B. Heyneman, D. Santos, and M. Cutkosky, "Smooth Vertical Surface Climbing With Directional Adhesion," Robotics, IEEE Transactions on, vol. 24, no. 1, pp. 6574, Feb.

[22] X.-H. Li, Y.-Q. Zhu, G.-Q. Huang, and S.-L. Nie, "Reaction thrust characteristics of high-pressure submerged water jet of cylinder nozzles," Journal of Chongqing University (English Edition). China, vol. 8, no. 1, pp. 63-69, 2009.

[23] G.-q. Huang, Y.-s. Yang, X.-h. Li, and Y.-q. Zhu, "Reaction thrust of water jet for conical nozzles," Journal of Shanghai University (English Edition), vol. 13, no. 4, pp. 305-310, 2009.

[24] S. Guo, X. Lin, and S. Hata, "A conceptual design of vectored waterjet propulsion system," in Mechatronics and Automation, 2009. ICMA 2009. International Conference on, pp. 1190-1195, IEEE, 2009. 